La agenda periodística en el aula. Relato de una experiencia didáctica en

Epidemiología y Salud Pública Básica Veterinaria.

Maria Cecilia Gortari, Estela Bonzo, Soledad Ceccarelli, Ninfa Rodríguez Mendoza, Karina Zubiri.

Trayectorias Universitarias, 5(8), e006, 2019

ISSN 2469-0090 | https://doi.org/10.24215/24690090e006

https://revistas.unlp.edu.ar/TrayectoriasUniversitarias

Universidad Nacional de La Plata

La Plata | Buenos Aires | Argentina

\title{
La agenda periodística en el aula. Relato de una experiencia didáctica en Epidemiología y Salud Pública Básica Veterinaria.
}

The news agenda in the classroom. Story of a experience in Epidemiology and Veterinary Basic Public Health

\author{
Soledad Ceccarelli | Ninfa Rodríguez Mendoza \\ https://orcid.org/0000-0002-9790-0157 https://orcid.org/0000-0001-7279-9593 \\ soledad.ceccarelli@gmail.com nrodriguez@untref.edu.ar
}

\author{
Maria Cecilia Gortari | Estela Bonzo |Karina Zubiri \\ https://orcid.org/0000-0002-4957-2531 https://orcid.org/0000-0003-0712-1289 https://orcid.org/0000-0003-3526-4869 \\ mcgortari@fcv.unlp.edu.ar ebonzo@fcv.unlp.edu.ar kzubiri@fcv.unlp.edu.ar \\ Facultad de Ciencias Veterinarias | Universidad Nacional de La Plata | Argentina
}

\section{RESUMEN}

Aunque la medicina veterinaria es uno de los pilares de la salud pública, esta relación no resulta tan evidente en los primeros años de la facultad. Esta situación, y las condiciones de adaptación por la que transitan los estudiantes hacen necesaria la intervención educativa para interesarlos en el área de salud pública. En este sentido, los medios de comunicación nos relatan hechos que involucran a los veterinarios en problemas actuales, reales y significativos de la práctica profesional, constituyéndose en una herramienta didáctica de enorme potencial formativo. Así, en Epidemiología y Salud Pública Básica incorporamos el uso de la prensa escrita en el aula. Este trabajo, da cuenta de dicha experiencia y profundiza sobre el potencial de la prensa escrita como recurso educativo.

Los resultados de la experiencia surgieron de una encuesta de opinión a los estudiantes y de un espacio de reflexión docente. La vinculación de la profesión con la actualidad, la motivación de estudiantes y docentes, y las potencialidades educativas de los medios de comunicación fueron lo más destacado. La "agenda periodística" en el aula, contribuyó a repensar la profesión en el área de Salud Pública y a la formación de profesionales más comprometidos con la sociedad.

\section{ABSTRACT}

Although veterinary medicine is one of the pillars of public health, this relationship is not so evident in the early years of the School of Science. This situation, and the conditions of adaptation which the students transit, make the educational intervention necessary so as to interest them in public health. In this sense, the media show us facts that involve veterinarians in current, real and significant problems about the professional practice, becoming a didactic tool with enormous training potential. Thus, in Epidemiology and Basic Public Health we incorporate the use of the written press in the classroom. This work reflects such experience and increases the potential of the written press as an educational resource. The results of the experience arose from an opinion poll from students and from a reflection space. The association of the profession with the present, the motivation of students and teachers, and the educational potential of the media were the highlights. The "journalistic agenda" in the classroom, helped to re-think the profession in the area of Public Health and the training of professionals more committed to society

\section{PALABRAS CLAVE}

Medios de comunicación, Educación, Propuesta didáctica, Medicina veterinaria.

\section{KEY WORDS}

Media, Education, Didactic proposal, Veterinary Medicine 


\section{INTRODUCCIÓN}

La medicina veterinaria es una profesión al servicio del ser humano por su participación en la prevención y control de enfermedades zoonóticas, en la producción de alimentos de calidad, en el saneamiento ambiental, en la salud animal. Por lo tanto, la formación en el área de salud pública es crucial para lograr una sociedad capaz de abordar los problemas sanitarios que plantea la interrelación animal-ser humano-ecosistemas (Vallat, 2013). Sin embargo, los estudiantes de veterinaria no sólo desconocen la importancia de su rol profesional en salud pública, sino que además suelen manifestar desinterés por las incumbencias en esta área. Esta situación que obedece a múltiples factores se refleja en encuestas, comentarios, en el diálogo con los alumnos y se repite en otras universidades del país y del extranjero (Pfuetzenreiter, 2006; Olascoaga and Olascoaga, 2012).

El curso de Epidemiología y Salud Pública corresponde al segundo año de la carrera de Medicina Veterinaria de la Universidad Nacional de La Plata (FCV-UNLP), circunstancia que agrega dificultades tales como el gran número de estudiantes, las características del plan vigente (correlatividades), el régimen de cursada vigente (formas de aprobación, presencialidad, espacio áulico) y el trabajo con estudiantes inexpertos en su rol de universitarios. El ingreso a la universidad implica un proceso complejo de cambio cultural en el que los estudiantes deben aprender el oficio de estudiante. Esta filiación a la universidad en los primeros años está marcada por una dificultad para la interpretación de textos, abstracción, relación e integración de conceptos, así como para la comunicación, adopción de estrategias, organización y técnicas de estudio (Casco, 2014). Esta situación y la tendencia a poner las responsabilidades de los fracasos académicos del lado de los estudiantes se ha naturalizado en la comunidad educativa. Del mismo modo, los docentes también hemos naturalizado las formas de enseñar y como consecuencia hemos obstaculizado la posibilidad de ampliar el repertorio de estrategias didácticas potencialmente útiles en el proceso de enseñanza aprendizaje.

En este contexto, nos propusimos trabajar estas dificultades a través de nuevas estrategias didácticas de intervención. En el trayecto, una conjunción del azar y la inquietud de algunos docentes nos llevó a in- 
corporar noticias periodísticas como recurso para motivar e implicar a los estudiantes en su rol profesional en la salud pública.

nos propusimos trabajar estas dificultades a través de nuevas estrategias didácticas de intervención. En el trayecto, una conjunción del azar y la inquietud de algunos docentes nos llevó a incorporar noticias periodísticas como recurso para motivar e implicar a los estudiantes en su rol profesional en la salud pública.

Los medios de comunicación masiva se presentan como una herramienta didáctica de enorme potencial para el proceso formativo (Blanco López, 2004; Hontangas, 2009; Sevillano García, 2015). Cotidianamente, los medios de comunicación ponen a nuestra disposición relatos de hechos o situaciones que involucran a los veterinarios en problemas actuales, reales y significativos de la práctica profesional. Estos relatos constituyen un recurso didáctico interesante cuya integración al sistema educativo es impulsado desde la UNESCO (2016). Los diarios y/o periódicos pueden ser considerados como el "libro del día" por la información que tienen sobre diferentes temas de la actualidad. Si bien hay grandes avances tecnológicos y mediáticos, la prensa escrita sigue siendo uno de los principales y más potentes medios de comunicación para la interpretación y valoración de la realidad (López, 2003; Vargas Benítez, 2009; Sevillano García, 2015). Su accesibilidad y diversidad informativa habilitan la posibilidad de fomentar el espíritu crítico, la reflexión, la autonomía respecto de la información recibida, la distinción entre hechos y opiniones y la formación de opinión sobre el tema, colaborando en la adquisición de aprendizajes reales y significativos (Hontangas, 2009). El objetivo de este trabajo es relatar y socializar la experiencia de la incorporación de la agenda periodística como recurso didáctico innovador en el curso de Epidemiología y Salud Pública Básica (FCV-UNLP).

\section{UN ACERCAMIENTO A LA PROFESIÓN A TRAVÉS DE LA AGENDA PERIODÍSTICA}

El curso de Epidemiología y Salud Pública Básica se separa en Actividades Presenciales Obligatorias (APOs) y en actividades virtuales complementarias. Es el primer contacto de los estudiantes con los contenidos del Núcleo de Medicina Preventiva y Salud Pública como área de incumbencia profesional. Sin embargo, en esta instancia de la carrera, la mayoría de los estudiantes desconoce aún las áreas de competencia profesional que define el Ministerio de Educación (Res. 1034, Ministerio de Educación, Ciencia y Tecnología) y priorizan Clínica o Producción según surge de encuestas anuales realizadas en la cursada. Frente a este escenario, la incorporación de artículos periodísticos que involucren a los veterinarios en el área de salud pública se presenta como una intervención didáctica con mucho potencial educativo. 
Pensamos en la prensa escrita, por ser un medio de comunicación estructuralmente más cercano al contexto educativo y a las posibilidades y necesidades de los usuarios: quién, con quién, cómo y para qué fines se va a utilizar (Sevillano García, 2015). Docentes y estudiantes aportaron espontáneamente noticias de interés sin limitaciones en relación a la fuente de la información (agencia informativa, corresponsal, servicios de documentación, etc.), al alcance de la información (municipal, provincial, nacional y/o internacional), o al género periodístico (noticia, entrevista, informe, reportaje, artículo de opinión, etc.).

Los objetivos formativos de la actividad fueron los siguientes:

- Fomentar la utilización de diversas fuentes informativas (no académicas) para la adquisición de nuevos conocimientos.

- Relacionar los contenidos del curso con la actualidad y la vida cotidiana

- Comprender la importancia de la prensa escrita como medio de difusión de la actualidad.

- Comprender, analizar y debatir sobre el vocabulario y la terminología científica utilizada en la prensa escrita.

- Fomentar el interés por la actualidad y promover una actitud crítica ante la información periodística.

- Visualizar y reforzar el rol profesional de los veterinarios en el área de Salud Pública.

El análisis de la noticia se inició a partir del título y lo que este sugiere y/o adelanta (alcance, intenciones, importancia, etc), su ubicación en el diario y/o periódico (sección, página), direccionando así un intercambio sobre la/las posibles interpretaciones del contenido, el uso de la terminología disciplinar o científica, la pertinencia para los veterinarios, el área de incumbencia involucrada, los posibles puntos de vista sobre la misma y los puntos de conexión con el curso. En algunos casos, dependiendo del momento y/o del docente, la presentación de la noticia fue más o menos elaborada (acompañada de diferentes versiones, con un seguimiento en el tiempo y acompañada de una serie de preguntas orientadoras).

Para identificar el impacto de esta innovación, por un lado se realizó el ejercicio del intercambio y reflexión crítica de las vivencias en el aula que experimentaron los docentes $y$, por otro lado, se consideró la opinión de los estudiantes, a través de las encuestas de fin de curso. 


\section{REFLEXIONES SOBRE LA INTERVENCIÓN Y LA DOCENCIA}

En este espacio, cada uno de los docentes compartió sus sensaciones, inquietudes y vivencias particulares. El ejercicio de reconstruir y compartir la propia experiencia se constituyó en un insumo para repensar y enriquecer la práctica docente, no solo para el propio docente sino para sus pares al generarse un espacio de reflexión colectiva (Edelstein, 2011). Como resultado de este trabajo participativo surgió una síntesis de los aspectos positivos de la experiencia:

- El carácter voluntario generó una motivación genuina en los estudiantes y un mayor compromiso al sentirse protagonistas de su propio aprendizaje.

- Las noticias resultaron ser textos "amigables" (cortos, interesantes, no académicos) constituyéndose en medios alternativos de acceso al conocimiento.

- El interés de los estudiantes retroalimentó la motivación de los docentes generando una continuidad en el aporte de noticias que trascendió, muchas veces, los tiempos formales del curso.

- La puesta en común de cada noticia permitió un intercambio en la clase que abarcó aspectos como el relato descriptivo, su valor como noticia, su pertinencia en el contexto del curso o de la profesión, el análisis de los contenidos, el uso y el significado del lenguaje disciplinar y nuevos interrogantes a la vez que facilitó una construcción colectiva de conocimiento a partir de lo que surgió en cada grupo.

- Además de lo específico y disciplinar, esta experiencia generó nuevas problematizaciones respecto de nuestra actividad como docentes y el reconocimiento de los espacios de discusión como herramienta fundamental para poner en cuestión nuestra práctica educativa.

\section{LA “AGENDA PERIODÍSTICA”}

La participación de los estudiantes fue más comprometida con dos tipos de noticias: 1) las que los involucra personalmente por ser ellos partícipes directos o indirectos del hecho y 2) las que se relacionaron con temas del curso, y por lo tanto pasaron a ser relevantes por su vínculo con la evaluación.

La incorporación de las noticias periodísticas trascendió el temario de cada APO conectándonos con hechos de la realidad local, regional y/o internacional.

\section{La incorporación de las noticias periodísticas trascendió el temario de cada APO conectándonos con hechos de la reali- dad local, regional y/o internacional.}

Algunos de los temas tratados fueron: tenencia responsable de mascotas, zoonosis, producción bovina, biotecnología, medio ambiente, contaminación ambiental, desastres naturales, cambio climático, con- 
trol de plagas, conservacionismo animal, salud pública, ética profesional. Particularmente hablamos de Chagas, hidatidosis, triquinosis, fiebre hemorrágica argentina, leptospirosis, rabia, gripe A, ébola, chikunguña, dengue, zika, leishmaniasis, brucelosis, aftosa, agrotóxicos, contaminación con mercurio, muerte de cóndores, erupción del volcán Puyehue, inundaciones, políticas públicas, vigilancia epidemiológica, uso responsable de antibióticos, diabetes, enfermedades transmitidas por alimentos, síndrome urémico hemolítico, botulismo.

Todos los temas fueron socializados a partir de un transparente virtual disponible en la plataforma moodle institucional () (Figura 1).

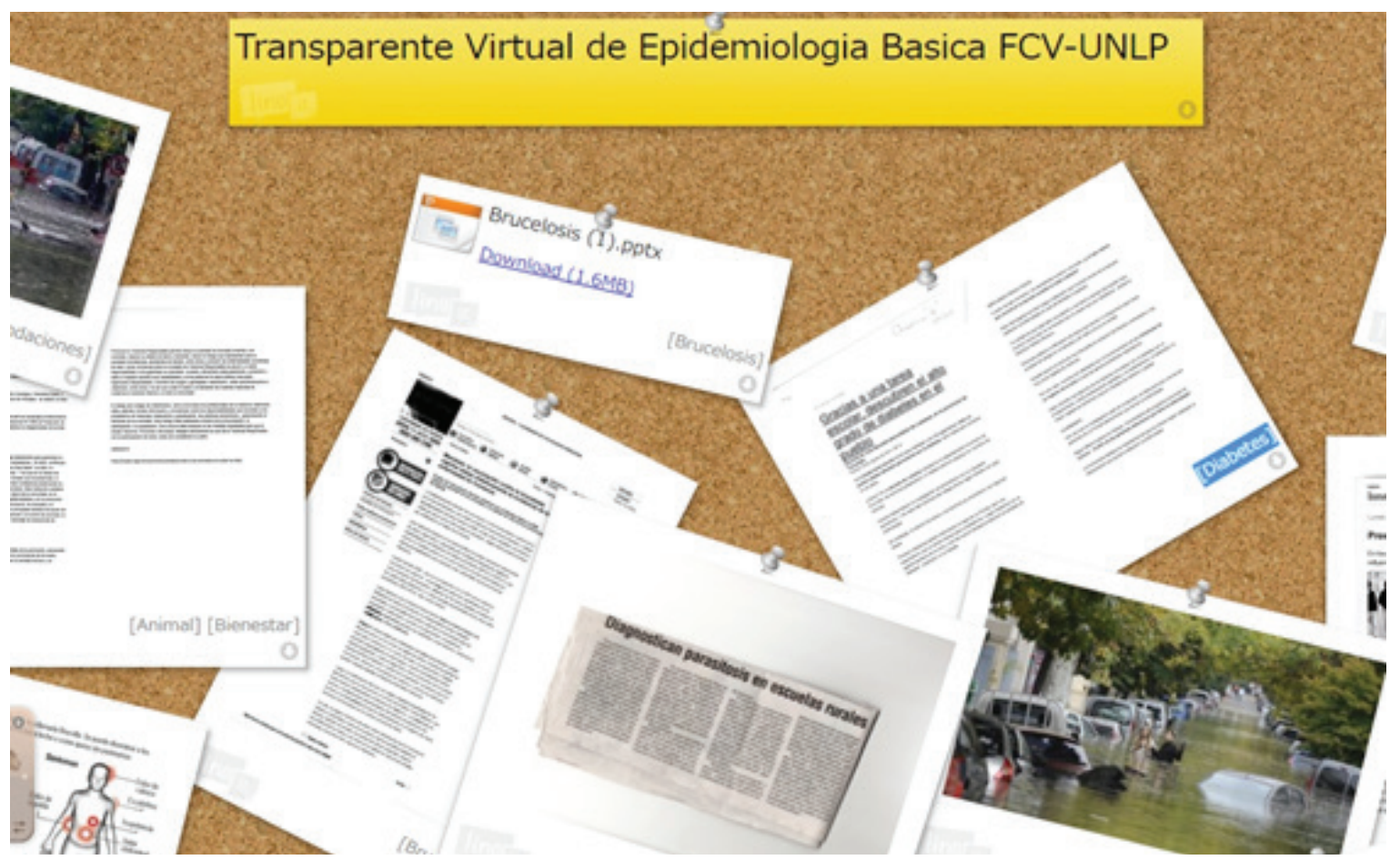

Figura 1: Transparente virtual disponible en la plataforma moodle institucional.

\section{COMENTARIOS Y APORTES DE LOS ESTUDIANTES}

En este punto hacemos mención a aquellos comentarios de los estudiantes que consideramos relevantes respecto de la estrategia didáctica implementada. Si bien es generalizado el apoyo a la continuidad de la actividad, consideramos importante destacar algunas valoraciones. Recuperamos comentarios que hacen referencia al vínculo con la realidad como "me pareció bueno porque relacionamos lo que aprendimos con la realidad" o "útil porque la relación entre el mundo real explicado desde el punto epidemiológico nos ayuda a ver el mundo y a ver los temas aplicados". Otros estudiantes ponen el acento en la profesión y la salud pública: "interesante ya que conocí la relación entre la salud pública y la veterinaria, su campo de estudio y demás cosas que hasta el momento no conocía". También se refieren a la integración del conocimiento: "Útil porque se pueden analizar los conceptos de las APOs de otra manera, que te genera más interés y además se relacio- 
nan con otras materias" y a otras formas de aprendizaje: "útil porque es una forma interactiva de adquirir conocimiento". Algo para destacar es la mención a la cuestión social:"Me pareció muy interesante ya que es una manera interesante de combinar los conocimientos adquiridos con cultura general y conciencia social", "muy útil porque te acerca a la realidad social lo visto en clase", a la vez que aparece su rol como comunicadores: "Hay muchos temas de la cursada relacionadas con la vida cotidiana y las noticias, muchas no las conocía o no estaba informada y me sirvió mucho para contarla a mi familia". Un/una estudiante recursante: "Soy recursante, me gustó mucho más esta cursada".

Los comentarios dan cuenta de que se enfrentaron a situaciones reales y complejas que implican un trabajo multidisciplinario y que difícilmente sean visualizadas en los programas de estudio. Esta información se presentó visualmente en una nube de palabras (http://www. nubedepalabras.es/) (Figura 2).

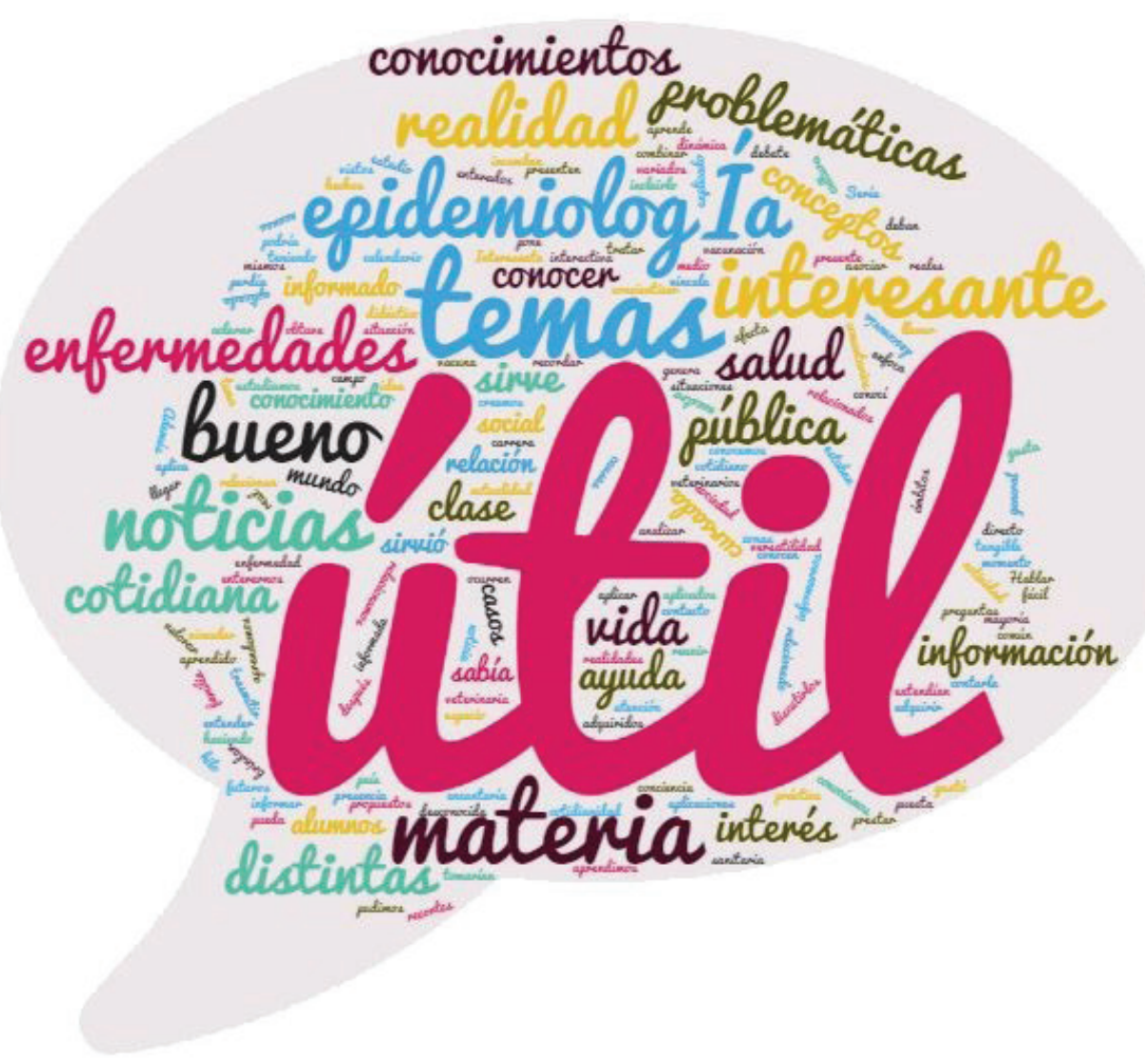

Figura 2. Representación visual (nube de palabras) de la opinión de los estudiantes respecto de la incorporación de la noticia como recurso didáctico.

Por otra parte, para algunos estudiantes el trabajo con las noticias tuvo poco o ningún valor. Sin embargo, no explican la causa de esa valoración y en algunos casos admiten desinterés y falta de participación: "No tengo computadora ni Internet y no creo muy interesante entrar para leer unos recortes periodísticos con datos epidemiológicos", "¡Perdón!, sé que tiene mucho que ver con la carrera, pero no me llamó mucho". Otros no entendieron el carácter voluntario y reclamaron "ique no sean obligatorias!". Pensamos que estos comentarios podrían rela- 
cionarse con un recorrido de educación tradicional que los condiciona a aprender de determinada manera y les impide visualizar la importancia de aprender a través de situaciones reales pero descontextualizadas del ámbito académico. También, hay que tener en cuenta que es difícil que los estudiantes valoren un conocimiento que no les es "útil" en este momento (no va a ser exigido ni evaluado en las instancias del curso) y hagan la siguiente consideración: "una pérdida de tiempo".

Como complemento se detallan los objetivos formativos alcanzados en base a los comentarios aportados por los estudiantes. Los mismos están acompañados por comentarios textuales tomados como ejemplo:

- Fomentar la utilización de diversas fuentes informativas (no académicas) para la adquisición de nuevos conocimientos.

«Útil porque es una forma interactiva de adquirir conocimientos»

- Relacionar los contenidos de la cursada con la realidad.

«Interesante ya que conocí la relación entre la salud pública y veterinaria, su campo de estudio y demás cosas que hasta el momento no conocía»

- Comprender la importancia de la prensa escrita como medio de difusión de la realidad.

«Útil porque la relación entre el mundo real explicado desde el punto de vista epidemiológico nos ayuda a ver el mundo y a ver los temas aplicados»

«Útil porque nos permite vincular conocimientos sobre las distintas enfermedades con conocimientos epidemiológicos y ver distintas problemáticas de la salud pública»

- Visualizar y reforzar el rol profesional de los veterinarios en el área de Salud Pública.

«Muy útil. Fue lo más relacionado con lo que me gusta de la carrera»

«Muy útil porque brindan un espacio de información y debate sobre distintas problemáticas que nos incumben como futuros veterinarios»

Algunos de los objetivos formativos descritos anteriormente como "Comprender, analizar y debatir sobre el vocabulario y la terminología científica utilizada en la prensa escrita" y "Fomentar el interés por la realidad y promover una actitud crítica ante la información periodística" no tuvieron vinculación directa con los comentarios de los estudiantes expresados en las encuestas. Sin embargo, fueron aspectos que se debatieron en la puesta en común de las noticias. 


\section{POTENCIALIDADES EN LA UTILIZACIÓN DE LA PRENSA}

La prensa escrita se ha utilizado como un recurso didáctico para la adquisición de información y conocimiento de la realidad social en diferentes disciplinas y desde diferentes perspectivas (López, 2003; Hontangas, 2009, Sevillano García, 2015). En Epidemiología y Salud Pública Básica, se utilizaron los medios de comunicación como una herramienta didáctica para motivar y promover la interacción con la realidad social utilizando los temas de actualidad vinculados con la profesión veterinaria y la salud pública. En este sentido, la realidad brindó la oportunidad de:

- Acompañar todo el desarrollo de los contenidos del curso siguiendo el tratamiento periodístico de un tema en el tiempo como por ejemplo sucedió con la epidemia/pandemia de ébola (2014-2015).

- Evaluar el tratamiento periodístico de los problemas relacionados con la salud, aunque hayan sucedido en un momento diferente al del desarrollo del curso como los brotes de dengue, chikungunya, etc.

- Visualizar la necesidad de la multidisciplinariedad para algunos hechos en los que están involucrados los animales y los veterinarios: la justicia, la economía, la medicina, etc.

- Por otro lado, mediante la lectura y el análisis de diarios y periódicos en el aula se desarrolla la capacidad para leer críticamente los mensajes publicados por los medios de comunicación. De este modo, se puede entender cómo:

- Construyen la realidad que informan

- Generan opinión pública

- Manejan la información al seleccionar no solo los contenidos que informan sino el cómo y el dónde los informan (uso de titulares, ilustraciones, fotos, mapas e infografía, la ubicación del texto en el diario, géneros, estilos, etc.)

- Manifiestan la subjetividad, los intereses y las tendencias ideológicas de la información.

- Ejercen influencia y determinan conductas sobre la comunidad.

- Tratan de maneras muy diversas una misma noticia o la prioridad de la información.

- Proponen o imponen agenda. 
En el contexto de la profesión y en salud pública los medios de comunicación tienen un rol de gran importancia en la generación de creencias y conductas que pueden afectar la salud individual y colectiva. Son muchos los mecanismos utilizados por los medios de comunicación masiva que vulneran el principio de "Una Salud" en íntima asociación con las industrias (agroalimentaria, farmacéutica, médica, etc.) y los gobiernos (Gérvas 2010; Feo Acevedo \& Feo Istúriz 2013; Granda Revilla 2015). Sin embargo, y sin dejar de reconocer los intereses que representan, es importante destacar su función educativa y su utilización para la promoción de la salud (Feo Acevedo \& Feo Istúriz 2013).

\section{CONCLUSIONES}

El recorrido de la experiencia que relatamos está marcado por la incorporación de hechos de la realidad cotidiana a través de la lectura de noticias periodísticas, utilizando como soporte la prensa escrita. La incorporación de la "agenda periodística" a Epidemiología y Salud Pública Básica acercó a los estudiantes a otras formas de adquirir conocimientos, al "hacer profesional" de los futuros veterinarios en el área de Salud Pública, así como a la formación de médicos veterinarios más comprometidos con la sociedad.

Estos recursos no tradicionales permitieron incorporar contenidos que si bien son parte de la currícula, no son "percibidos" por los alumnos como parte del rol del veterinario y condicionaron el abordaje de temas de actualidad vinculados a la profesión, que de no ser así, no hubieran sido incluidos en el desarrollo del curso.

Estos recursos no tradicionales permitieron incorporar contenidos que si bien son parte de la currícula, no son "percibidos" por los alumnos como parte del rol del veterinario y condicionaron el abordaje de temas de actualidad vinculados a la profesión, que de no ser así, no hubieran sido incluidos en el desarrollo del curso.

Respecto de las actividades potenciales de la prensa escrita, se han mencionado algunas posibilidades de uso como recurso educativo en cuestiones vinculadas con la veterinaria y la salud pública. Sin embargo, el uso de los medios masivos de comunicación ofrece múltiples posibilidades para trabajar en los procesos de enseñanza-aprendizaje en cualquier disciplina y desde diferentes perspectivas.

La incorporación de los medios de comunicación en el aula constituye un importante recurso pedagógico en el proceso de enseñanza-aprendizaje. La multiplicidad de usos que permite la diversidad de información proporcionada por los medios, constituye un importante recurso para vincular a los alumnos con la realidad social y profesional en la que viven.

Agradecimientos y reconocimientos

Al Médico Veterinario Raúl Müller y al estudiante Exequiel Ranieri por su colaboración 


\section{BIBLIOGRAFIA}

Blanco Lopez, A. (2004). "Relaciones entre la educación científica y la divulgación de la ciencia". Revista Eureka sobre enseñanza y divulgación de las ciencias, 1(2), pp. 70-86.

Casco, M. (2014). "Afiliación intelectual y escritura en la universidad: Un estudio de caso sobre la perspectiva docente". Bellaterra Journal of Teaching \& Learning Language \& Literatura, 7(2), pp.34-53.

Edelstein, G. (2011). “Formar y formarse en la enseñanza”. Buenos Aires: Paidós

Feo Acevedo, C. \& Feo Istúriz, O. (2013).“"Impacto de los medios de comunicación en la salud pública". Saúde em Debate, 37(96), pp.84-95.

Gérvas, J. (2010). "El abordaje de la pandemia de gripe A desde la perspectiva de los profesionales sanitarios". Atención Primaria, 42(11), pp.541-542.

Granda Revilla, J. (2015). "Cómo aprender de los errores. Comunicación en crisis sanitarias". Mètode, 0(88), pp.69-73.

Hontangas, N. A. (2009). “Los medios de comunicación, desde su vertiente didáctica, dentro de la Universidad". Prisma Social, (3), pp. 1-21.

López, A. P. L. (2003). “La prensa escrita, recurso didáctico”. Edited by Ministerio de Educación: CIDE. Madrid.

Ministerio de Educación, Ciencia y Tecnología. http://www.bnm.me.gov.ar/ giga1/normas/RM_1034-05.pdf

Olascoaga, C. and Olascoaga, C. (2012). "Conferencia Acción del veterinario ante la biodiversidad de las Ciencias Veterinarias", pp. 259-277.

Pfuetzenreiter, M. R. (2006). “La Enseñanza de la Salud Pública en Escuelas de Medicina Veterinaria en Brasil". Revista Electrónica de Veterinaria, 7, pp. 1-14.

Sevillano García, M.L (2015). "La enseñanza en todos los niveles formativos renovada con la actualidad de la prensa escrita". Aularia 1, pp.031-038.

UNESCO (2016). “"Enseñar para los medios, o medios para enseñar?".http://www.unesco.org/new/es/media-services/single-view/news/media_and_information_literacy/

Vallat, B. (2013). “El concepto "Una sola salud": enfoque de la OIE". Boletín de la Organización Mundial de Sanidad Animal, OIE, 2013-1, pp. 2-4.

Vargas Benitez, A. (2009). “El uso didáctico de la prensa en el aula y su aportación a las competencias básicas". Revista Digital del Centro del Profesorado de Alcalá de Guadaira 1 (4),pp. 1-17. 